\title{
A pilot study for exploring blood spot anti-mullerian hormone for population-based adolescent reproductive health research
}

\author{
Kelli S Hall ${ }^{1}$, Shelby T Rentmeester ${ }^{*}$, Yuan Zhao $^{2}$, Allison N Hankus ${ }^{3}$, Yidan Pei ${ }^{3}$, Halley EM Riley ${ }^{1}$, Candace McCloud ${ }^{1}$ and Bradley D \\ Pearce $^{3}$ \\ ${ }^{1}$ Department of Behavioral Sciences and Health Education, Emory University Rollins School of Public Health, 1518 Clifton Rd NE, Atlanta, GA 30322, USA \\ ${ }^{2}$ Department of Biostatistics, Emory University Rollins School of Public Health, 1518 Clifton Rd NE, Atlanta, GA 30322, USA \\ ${ }^{3}$ Department of Epidemiology, Emory University Rollins School of Public Health, 1518 Clifton Rd NE, Atlanta, GA 30322, USA
}

\begin{abstract}
Introduction and objective: Studies of Anti-Müllerian Hormone (AMH) rely upon serum measures and clinical samples of older reproductive-aged women intended/attempting pregnancy, with known fertility issues or medical morbidities. We explored the utility of minimally invasive AMH as a measure of fecundability in population-based reproductive health research.

Methods: We analyzed baseline data from 191 participants in a pilot, longitudinal cohort study, the Young Women's Stress Study. Using an integrated biosocial design, we collected interviewer-administered surveys on demographic, psychosocial, health, and method feasibility/acceptability information and finger-stick capillary dried blood spots (DBS). We used descriptive and bivariate statistics (correlation, T-tests, ANOVA) to estimate method feasibility/acceptability and unadjusted AMH mean concentrations overall and across sociodemographic, reproductive, and health covariates.

Results: AMH concentrations ranged from 1.02 to $22.23 \mathrm{ng} / \mathrm{mL}$, with a mean of $5.66 \mathrm{ng} / \mathrm{mL}$. AMH concentrations were associated with current hormonal contraceptive use, menstrual cycle frequency, and irregular menstrual patterns, but not with other known correlates. Most participants stated the DBS method was comfortable (81\%) and would be likely to provide it again (88\%).

Conclusions: While these pilot data suggest AMH fell within normal range and our DBS methods were acceptable/feasible, the broader question of its usefulness for population reproductive health research remains unanswered. Larger, longitudinal studies are needed to validate AMH against time-to-pregnancy and gold standard measures in young healthy samples and across different sociodemographic groups. Public health and social scientists should consider the resource costs of AMH, ethical issues, and risks of (over)interpretation, with a reproductive justice and human rights frame in mind.
\end{abstract}

\section{Background}

While the proximate behavioral determinants of unintended pregnancy (i.e. sex and contraception) have been the focus of family planning research for more than three decades [1-4], the intersecting biological and social pathways leading to pregnancy during adolescence and young adulthood have been understudied $[5,6]$. Current public health priorities emphasize the need to integrate biology and social context in research in order to understand underlying mechanisms of health disparities [7-9]. Yet, biological processes of health have been relatively ignored in social science studies of reproductive health. Landmark population-based studies that have addressed young women's reproduction have not included biomarkers of fertility or biomarkers at all $[10,11]$.

Research in reproductive endocrinology has long studied the fertility of women later in the reproductive life course, and various measures of infertility have been widely tested and used $[12,13]$. Biomarkers of fertility often relied upon in clinical studies, such as antral follicle count (ultrasonography of follicle number) and neuroendocrine steroid hormones of the hypothalamus-pituitary axis (follicle-stimulating hormone (FSH), luteinizing hormone (LH) and estrogen), have features which limit their feasibility and utility for population research. Invasiveness, significant cost, necessary tightly controlled conditions, intensive data collection procedures, burdensome follow-up schedules, substantial inter-patient variability and intra-cycle fluctuation, and limited predictive value are among the limitations of these common fertility measures $[12,13]$.

Anti-Mullerian hormone (AMH), a biomarker of ovarian reserve and measure of fertility used in clinical studies, has unique features that may hold potential for population-based and social science reproductive health research [13-27]. A glycoprotein dimer produced by granulosa cells from small pre-antral and antral follicles in the ovary, AMH inhibits recruitment of primordial follicles in the pool of growing follicles $[13,14]$. AMH is a useful ovarian reserve measure because it plays an important role in early-stage follicle development (Figure 1) [16], fluctuates negligibly during the menstrual cycle and can be easily quantified in serum $[13,14]$. A few recent studies suggest

${ }^{\star}$ Correspondence to: Shelby T. Rentmeester, Department of Behavioral Sciences and Health Education, Emory University Rollins School of Public Health, 1518 Clifton Rd NE, Atlanta, GA 30322, USA, Tel: 470-217-3230; E-mail: shelby. rentmeester@emory.edu

Key words: adolescence; Anti-Müllerian hormone; public health research unintended pregnancy; young women

Received: January 16, 2020; Accepted: January 31, 2020; Published: February 03,2020 


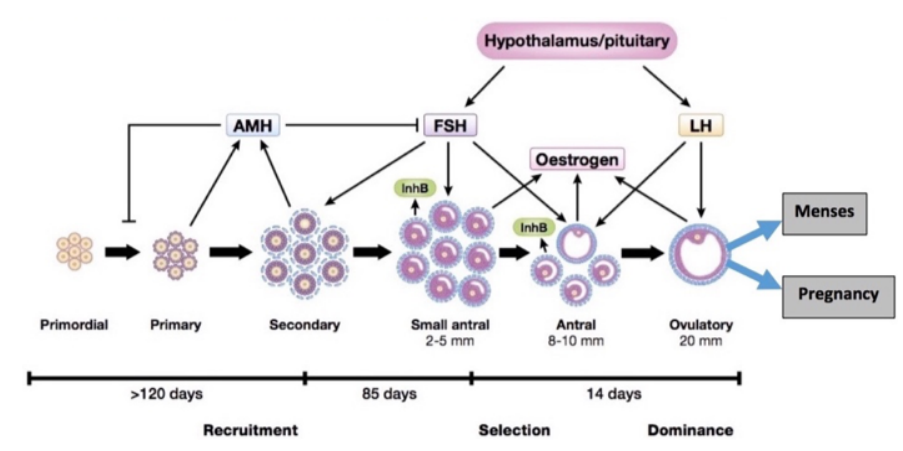

Figure 1. Aim 1 biomarkers to measure different stages of folliculogenesis and fecundability [16].

that minimally invasive dried blood spot AMH assays are reliable and comparable to serum samples [15].

AMH levels are believed to be age-dependent, appearing stable through adolescence, peaking at age 25 , and then slowly decreasing until becoming undetectable at menopause when the primordial follicle stock is exhausted [18]. The significance of both low and high AMH levels in regularly menstruating young women, however, is unclear [18-22]. Rather, the majority of AMH research has measured ovarian function in cases of menopausal transitions, diagnosed or observed subfecundity, in vitro fertilization, cancer treatment, and specific diseases [13-24]. The few AMH studies that have included adolescent and young adult women have similarly focused on specific disorders (cancer, Turner Syndrome, polycystic ovarian syndrome, precocious puberty, Fragile X), clinical settings, and venipuncture alone [18-22]. More recent $\mathrm{AMH}$ studies of health(ier) women earlier in the reproductive life course (30-45 years) have been limited by predominantly White homogenous samples of women planning conception, recruited from clinical settings, and reliance upon serum AMH measures [24].

Research is needed to understand whether a minimally invasive $\mathrm{AMH}$ bioassay is a useful reproductive health indicator for young, healthy, racially diverse women not actively seeking or even intending to avoid pregnancy and in community settings. We sought to explore the utility of blood spot AMH as a potential measure of fecundability for population-based reproductive health and family planning research. We also tested differences in blood spot AMH levels across racial/ethnic and young age groups and to examine physical, mental, reproductive, and behavioral health correlates of AMH among our young, healthy cohort.

\section{Materials and methods}

Data are drawn from a subsample of the Young Women's Stress Study, a community-based longitudinal cohort pilot study of 199 females aged 15-24 years recruited from a large metropolitan area in the South-eastern United States. Interested potential participants were recruited through various methods including advertising on social media platforms (31\%) and in person at community locations (24\%), such as relevant teen and health-based community organizations, shopping centres, and local events, and snowball sampling methods (25\%). Participants were eligible if they were English speaking and had internet access. Young women were excluded if they had not yet experienced menarche, were pregnant at baseline, or had history of primary amenorrhea, known diagnosis of an ovarian disease (eg. Polycystic Ovarian Syndrome-PCOS) or cancer and/or exposure to gonadotoxic therapies. Those enrolled in the study received a $\$ 50$ gift card for completing the baseline and yearly appointments and $\$ 10$ for every two online surveys they completed for 11 months. The Emory University Institutional Review Board reviewed and approved the study materials and procedures prior to initiation. All study participants provided informed consent (or assent and parental consent when applicable) before enrolment in the study.

\section{Biological data collection}

Biological data was collected from each participant at the start of the baseline appointment to measure a series of bioassays for AMH and stress biomarkers. Finger-stick dried blood spots (DBS) via drops of whole capillary blood collected on filter paper were collected from each. Among the original cohort of approximately 200 young women, nine of the enrolled refused or could not partake in the DBS data collection. Information regarding reasons for refusal was collected. Finger-stick capillary blood samples were collected on blood collection filter paper (GE Healthcare - Whatman 903 Protein Saver, Chicago, IL, USA) by delivering a controlled, uniformed puncture with a sterile, disposable micro-lancet (McKesson \#927257 and 927258, Irving, TX, USA). After wiping away the initial drop of blood with sterile gauze, up to five drops of whole blood were applied to the filter paper. The samples were then allowed to dry at room temperature and stored frozen at $-20^{\circ} \mathrm{C}$ at an on-site lab facility.

In addition to DBS data, we also collected information regarding anthropometric measures of health (blood pressure, height, weight, waist circumference) and a hair sample, before initiating an in-depth interviewer administered psychosocial survey. Methods and data regarding the stress measures are reported elsewhere.

\section{Extraction from DBS}

AMH DBS extraction followed the modified extraction protocol from the AMH DBS ELISA kit (Ansh Labs, \#AL-129, Webster, TX, USA) [25-28]. Two discs of each sample were punched out directly into one well of a 24 well (Cat \#25820) plate using a $7.9 \mathrm{~mm}$ puncher (McGill Metal Product, \#MCG52300C, FL, USA). Discs were eluted with $450 \mu \mathrm{L}$ bovine serum albumin (BSA) based extraction buffer with a non-mercury preservative (Ansh Labs, \#ASB205, Webster, TX, USA) and incubated with shaking $(200 \mathrm{rpm})$ at room temperature (RT, $23 \pm$ $2^{\circ} \mathrm{C}$ ) for $1 \mathrm{hr}$. Liquid was transferred to cryotubes and stored at $-80^{\circ} \mathrm{C}$ until needed for the assay.

\section{AMH assay}

Quantitation of the AMH concentration was determined with the AMH ELISA kit (Ansh Labs, \#AL-105, Webster, TX, USA) and the modified protocol from the DBS ELISA kit mentioned above. Each assay provided low- and high-level controls of known concentration for quality control. Calibrators $(100 \mu \mathrm{L})$ and Assay Controls $(100 \mu \mathrm{L})$ provided by the kit were added in duplicate to corresponding wells of the labelled microtitration strip followed by $50 \mu \mathrm{L}$ Assay Buffer. After the addition of $150 \mu \mathrm{L} /$ well of Samples and the Sample Control in duplicate to the remaining wells, the plate was incubated for $3 \mathrm{hrs}$ (700 rpm, RT), rinsed 5 times $(350 \mu \mathrm{L} /$ well) with the Wash Solution (supplied by the AMH ELISA kit), followed by Antibody-Biotin Conjugate RTU $(100 \mu \mathrm{L})$ and incubation for $1 \mathrm{hr}, 700 \mathrm{rpm}, \mathrm{RT})$. After washing the plate, the Streptavidin-Enzyme Conjugate-RTU $(100 \mu \mathrm{L})$ was then added, incubated for $30 \mathrm{mins}, 700 \mathrm{rpm}, \mathrm{RT}$ ), and then washed as previously. Addition of TMB chromogen solution and incubation at $700 \mathrm{rpm}$, RT for 8-12 mins was followed by Stop Solution and reading the absorbance at $450 \mathrm{~nm}$ and $630 \mathrm{~nm}$. AMH concentration was calculated from the standard curve with the Equation $\log (\mathrm{Y})=$ 
$\mathrm{D}^{\star} \log (\mathrm{X})^{\wedge} 3+\mathrm{C}^{\star} \log (\mathrm{X})^{\wedge} 2+\mathrm{B}^{\star} \log (\mathrm{X})+\mathrm{A}$. All samples fell within the detection range and controls were within acceptable ranges. The final concentration was obtained by multiplying the calculated concentration from the fitted curve by 16 to account for volume of plasma on blood spots according to the suggestion of the manufacturer.

\section{Assay, intra-assay, and inter-assay control}

The concentration of the low and high controls of all assays fell within the concentration range established for the kit. The intra-assay CV of all unknowns was $9.18 \%$. Low and high assay controls supplied by the kit were used as the inter-assay control and their CVs were $8.73 \%$ and $9.14 \%$ respectively.

\section{Psychosocial interviewer-administered surveys}

In addition to biological data collection, the baseline comprehensive psychosocial survey measured various aspects of mental, physical, and reproductive health along with factors that contribute to a participant's social and physical context. The interviewer-administered survey comprised of over 850 questions, including sections on sociodemographic information, physical and mental health history, status, health service use, and behaviours, sexual and reproductive histories, including menstrual, contraceptive and pregnancy histories, and social and environmental contexts. We then asked participants to complete brief web-based monthly surveys for one year that assessed abbreviated versions of the above-described measures in order to capture dynamic, time-varying health, social and reproductive experiences across the study period. When available, the survey consisted of validates scales or were adapted from other surveillance and longitudinal datasets (i.e. Youth Risk Behaviour Surveillance System; Add Health) for the baseline, monthly, and yearly surveys. Study data were collected and managed using REDCap electronic data capture tools hosted at Emory University [29,30].

\section{Correlate measures}

Our psychosocial and health survey included a number of covariate measures that we hypothesized correlate with AMH levels. These measures were determined a priori based on previous research $[24,26]$. For the present analysis we focus on baseline correlate measures since they were collected at the time of AMH. Sociodemographic information includes age, race, and educational attainment. Physical health covariates include Body Mass Index (BMI), current smoking status, and history of chronic medical conditions. The chronic medical conditions assessed include asthma, arthritis, cancer, diabetes, heart disease, high blood pressure, high cholesterol, skin cancer, substance abuse disorder and psychological stress. Gynecological health covariates include age at menarche, menstrual cycle length, history of irregular menstrual periods, history of pregnancy and gynecological disorders including ovarian cysts and vaginitis.

\section{Feasibility and acceptability measures}

Our baseline and one-year follow up interviewer-administered surveys asked a series of feasibility and acceptability items related to our integrated biosocial measurement approach. Relevant to our DBS $\mathrm{AMH}$ data collection procedures, a sub-set of 58 participants were asked the following items at the annual follow-up: 1) "At your baseline appointment, how comfortable or uncomfortable was it to give a blood sample," on a 4-point likert scale ranging from very comfortable to very uncomfortable; and 2) "How likely or unlikely would you have given another blood sample at your yearly appointment," on a 5-point likert scale ranging from very likely to very unlikely.

\section{Statistical analysis}

We used univariate statistics to estimate distributions of mean $\mathrm{AMH}$ concentrations $(\mathrm{ng} / \mathrm{ml})$ among the sample and unadjusted bivariate tests (correlation, independent sample T-tests, and ANOVA) to compare AMH mean concentrations across different demographic, social, reproductive and general health covariates. We used SAS 9.4 for all analyses.

\section{Results}

Characteristics of our analytic sample $(n=191)$ are presented in table 1 . The mean age of participants was 20.3 years. The majority self-identified as Black/African American (40\%) or Non-Hispanic White $(26 \%)$ race/ethnicity. A third of participants were still in high school (35\%) and a quarter reported annual parental income levels $<\$ 45,000$ (26\%). Approximately two-thirds had ever used a hormonal contraception (63\%), with one-fifth currently using a hormonal method (20\%). The average age of menarche was 12 years and most participants reported 28-32 days menstrual cycles. Seventeen percent had ever been pregnant. Histories of gynecological or chronic medical conditions or of tobacco use were rare.

The distribution of AMH ranged from 1.02 to 22.23, with a mean concentration of $5.66 \mathrm{ng} / \mathrm{mL}$ (Figure 2). In bivariate analyses exploring AMH covariates, only current hormonal contraceptive use, menstrual cycle frequency, and irregular menstrual patterns were associated with mean AMH concentrations (Table 1). AMH concentrations $(\mathrm{ng} / \mathrm{ml})$ were higher among non-users of contraception $(\mathrm{m}=6.184)$ vs. users $(\mathrm{m}=4.914 ; \mathrm{p}=0.03)$, among participants with menstrual cycles longer than 32 days $(m=7.073)$ vs. those with cycles $28-32$ days $(m=5.378)$ or $<28$ days $(\mathrm{m}=4.346 ; \mathrm{p}=0.007)$, and among those with a history of irregular menses $(\mathrm{m}=6.092)$ vs. those without irregular menstrual histories $(\mathrm{m}=4.585 ; \mathrm{p}=0.018)$. Other known AMH covariates were not associated with AMH mean concentrations among this sample (Table 1) $[31,32]$.

Regarding feasibility and acceptability of our biological data collection methods $(n=58), 81 \%$ stated it was very or somewhat comfortable giving a blood sample at baseline and $88 \%$ stated they would have been very or somewhat likely to give a blood sample at the yearly appointment. Some reasons participants did not provide a blood sample at the baseline appointment include participants feeling

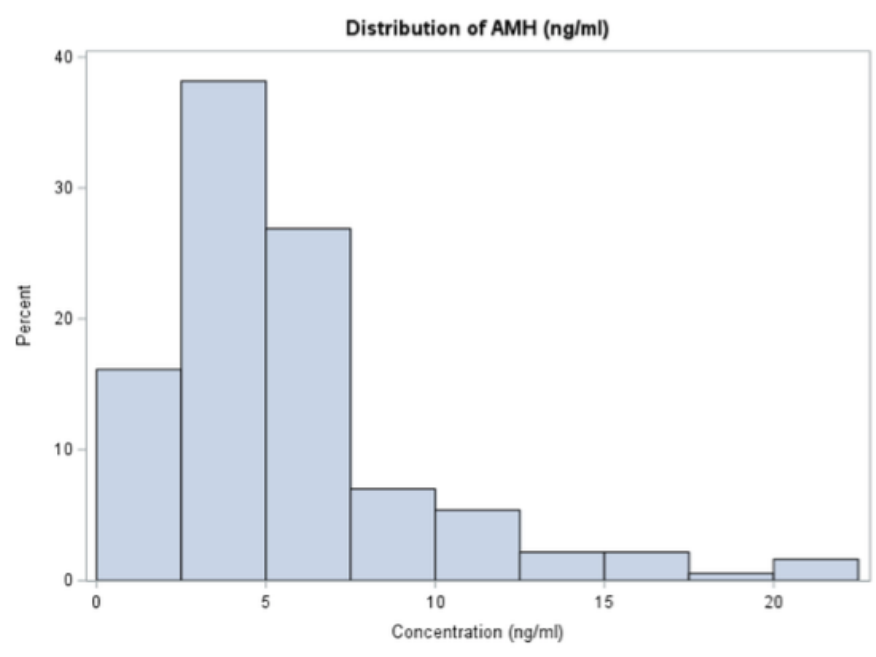

Figure 2. Mean anti-mullerian hormone concentration distribution (N=191). 
Hall KS (2020) A pilot study for exploring blood spot anti-mullerian hormone for population-based adolescent reproductive health research

Table 1. Characteristics of the sample and mean AMH concentrations across covariate groups. Full sample ( $\mathrm{n}=191)$ descriptive results are presented as frequencies and proportions or means with standard deviations. AMH concentrations are presented as means with standard deviations and mean differences tested across covariate groups with unadjusted bivariate correlations or ANOVA. *Alpha $<0.05$ considered significant for bivariate tests across sociodemographic and reproductive history groups.

\begin{tabular}{|c|c|c|c|}
\hline $\begin{array}{l}\mathrm{N}=191 \\
\text { Covariates }\end{array}$ & $\begin{array}{l}\text { Sample Characteristics } \\
\text { M (SD) or \# (\%) }\end{array}$ & $\begin{array}{l}\text { AMH Mean Concentrations (SD) or } \\
\text { Correlation }\end{array}$ & P-value \\
\hline Age (continuous) & $20.3(2.8)$ & 0.02 & 0.82 \\
\hline $\begin{array}{l}\text { Age Categories (years) } \\
15-17 \\
18-20 \\
21-24\end{array}$ & $\begin{array}{l}35(18.3) \\
60(31.4) \\
96(50.3)\end{array}$ & $\begin{array}{l}4.85(2.79) \\
6.27(4.55) \\
5.59(3.79)\end{array}$ & 0.23 \\
\hline BMI (continuous) & $25.9(7.2)$ & -0.01 & 0.89 \\
\hline $\begin{array}{l}\text { BMI Categories } \\
\text { Normal }(<25) \\
\text { Overweight }(25-29.9) \\
\text { Obese }(>=30.0)\end{array}$ & $\begin{array}{l}110(57.6) \\
38(19.9) \\
43(22.5)\end{array}$ & $\begin{array}{l}5.77(3.83) \\
5.52(3.94) \\
5.52(4.15)\end{array}$ & 0.92 \\
\hline $\begin{array}{l}\text { Race } \\
\text { Non-Hispanic White } \\
\text { Black or African American } \\
\text { Hispanic or Latino } \\
\text { Asian or Pacific Islander } \\
\text { Other }\end{array}$ & $\begin{array}{l}49(25.7) \\
76(39.8) \\
22(11.5) \\
17(8.9) \\
27(14.1)\end{array}$ & $\begin{array}{l}5.60(3.88) \\
5.77(3.81) \\
6.59(5.44) \\
4.63(1.72) \\
5.43(3.85)\end{array}$ & 0.64 \\
\hline $\begin{array}{l}\text { Education } \\
\text { High School Degree or Less } \\
\text { Some College } \\
\text { College Degree or More }\end{array}$ & $\begin{array}{l}67(35.1) \\
69(36.1) \\
55(28.8)\end{array}$ & $\begin{array}{l}5.92(4.14) \\
5.78(3.85) \\
5.35(3.80)\end{array}$ & 0.70 \\
\hline $\begin{array}{l}\text { Parental Income } \\
<14,999 \\
15,000 \text { to } 44,999 \\
45,000-74,999 \\
>75,000\end{array}$ & $\begin{array}{l}15(7.9) \\
35(18.4) \\
36(19.0) \\
66(34.7)\end{array}$ & $\begin{array}{l}6.03(4.10) \\
6.21(4.00) \\
6.37(4.58) \\
5.34(3.89)\end{array}$ & 0.62 \\
\hline $\begin{array}{l}\text { Own Income } \\
<1,000 \\
1,000-9,999 \\
10,000-24,999 \\
>=25,000\end{array}$ & $\begin{array}{l}55(29.3) \\
70(37.2) \\
34(18.1) \\
29(15.4)\end{array}$ & $\begin{array}{l}4.98(3.45) \\
5.94(3.69) \\
6.36(4.37) \\
5.71(4.73)\end{array}$ & 0.40 \\
\hline $\begin{array}{l}\text { Ever Hormonal Contraceptive Use } \\
\text { No } \\
\text { Yes }\end{array}$ & $\begin{array}{c}71(37.2) \\
120(62.8)\end{array}$ & $\begin{array}{l}5.16(2.88) \\
5.97(4.39)\end{array}$ & 0.18 \\
\hline $\begin{array}{l}\text { Current Hormonal Contraceptive Use } \\
\text { No } \\
\text { Yes }\end{array}$ & $\begin{array}{l}114(59.7) \\
77(20.3)\end{array}$ & $\begin{array}{l}6.18(4.34) \\
4.91(3.04)\end{array}$ & $0.03 *$ \\
\hline $\begin{array}{l}\text { History of Pregnancy } \\
\text { No } \\
\text { Yes }\end{array}$ & $\begin{array}{l}159(83.3) \\
32(16.8)\end{array}$ & $\begin{array}{l}5.68(3.84) \\
5.57(4.29)\end{array}$ & 0.89 \\
\hline $\begin{array}{l}\text { Menstrual Cycle Frequency } \\
\text { Less than } 28 \text { days } \\
\text { 28-32 Days } \\
\text { More than } 32 \text { days }\end{array}$ & $\begin{array}{c}34(18.2) \\
116(62.0) \\
37(19.8)\end{array}$ & $\begin{array}{l}4.35(2.23) \\
5.38(3.37) \\
7.07(5.00)\end{array}$ & $0.01 *$ \\
\hline $\begin{array}{l}\text { Irregular Menses Ever } \\
\text { No } \\
\text { Yes }\end{array}$ & $\begin{array}{c}54(28.3) \\
137(71.7)\end{array}$ & $\begin{array}{l}4.59(2.99) \\
6.09(4.140)\end{array}$ & $0.02 *$ \\
\hline Age at Menarche (continuous) & $12.2(1.6)$ & 0.11 & 0.15 \\
\hline Gynecological Conditions: & & & \\
\hline $\begin{array}{l}\text { Ovarian Cysts } \\
\text { No } \\
\text { Yes }\end{array}$ & $\begin{array}{l}178(93.2) \\
13(6.8)\end{array}$ & $\begin{array}{l}5.69(4.18) \\
5.39(3.89)\end{array}$ & 0.79 \\
\hline $\begin{array}{l}\text { Vaginitis } \\
\text { No } \\
\text { Yes }\end{array}$ & $\begin{array}{l}158(82.7) \\
33(17.3)\end{array}$ & $\begin{array}{l}5.47(3.69) \\
6.61(4.75)\end{array}$ & 0.14 \\
\hline $\begin{array}{l}\text { Currently Smoking } \\
\text { No } \\
\text { Yes }\end{array}$ & $\begin{array}{l}170(89.0) \\
21(11.0)\end{array}$ & $\begin{array}{l}5.61(3.96) \\
6.07(3.50)\end{array}$ & 0.61 \\
\hline $\begin{array}{l}\text { Environmental Exposure Assessment } \\
\text { (Continuous Scale, range 0-21) }\end{array}$ & $10.3(2.8)$ & -0.05 & 0.54 \\
\hline $\begin{array}{l}\text { Any Chronic Medical Conditions }{ }^{b} \\
\text { No } \\
\text { Yes }\end{array}$ & $\begin{array}{l}137(71.7) \\
54(28.3)\end{array}$ & $\begin{array}{l}5.8(4.1) \\
5.3(3.4)\end{array}$ & 0.38 \\
\hline Psychological Stress (Continuous Scale, range 0-56) ${ }^{\mathrm{c}}$ & $39.4(8.4)$ & 0.02 & 0.78 \\
\hline
\end{tabular}

Note: 38 responded "Don't Know" to parental income

aEnvironmental Exposure Assessment [30] is additive index score of 21 different types of environmental exposures known to be associated with fertility and correlates of AMH.

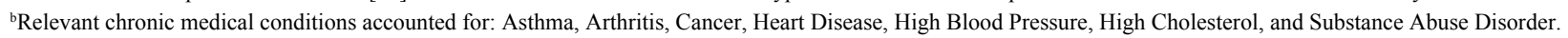

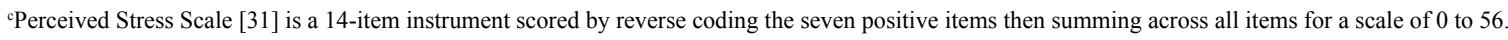


faint, unable to obtain enough sample from a second finger prick, or participant or parent not consenting to the sample.

\section{Conclusion}

In this analysis of data from our original biosocial pilot study, we sought to explore the utility of blood spot AMH as measure for population-based adolescent reproductive health and family planning research. Most other existing studies on AMH have used serum measures and clinical-based samples of older reproductive aged women intended or attempting pregnancy, with known fertility issues or medical morbidities. In the Time to Conceive study of 98 predominantly white, educated women ages 30-42 years recruited from community settings who were intending immediate pregnancy, low serum AMH levels were found to predict reduced fecundability $[23,24]$. On the other hand, a larger follow-up replication study by Steiner et al (2017) reported inconsistent findings on AMH levels and time to pregnancy, such that the authors questioned the usefulness of AMH as a reproductive function measure in adult community-based samples [24]. As to whether AMH levels vary across different racial/ethnic groups, the majority of AMH research has been conducted on White women and European cohorts [18-21]. Studies including racially diverse samples in the U.S. have focused on women of mid to late childbearing years [25-27]. Several prospective studies in specific contexts (e.g. HIV) found greater age-related declines in $\mathrm{AMH}$ over time among African American women compared to White women [27]. A cross-sectional study of a community-based multi-ethnic sample of 947 healthy, regularly cycling women found African American adult women ( $>25$ years) had lower AMH levels than White women [26]. None of these studies included adolescents or young adults. The only study of which we are aware to focus on a younger diverse but clinically-based sample (10-21 years), in fact, reported modestly higher serum AMH levels among African Americans (3.25 ng/mL) compared to Whites (3.19ng/ $\mathrm{ml}$ ), which were statistically similar in age-controlled models [25].

Our study builds upon this work by: 1) applying a minimally invasive, sensitive measure of ovarian reserve to community-based "real world" settings [13-16]; 2) focusing on adolescent and young adult women - a group that has been understudied in AMH research and in regards to the biosocial pathways to fertility and yet for whom the consequences of unintended pregnancy are most severe $[3,33,34]$; 3) using a life course theoretical approach that considers AMH during the critical developmental period of adolescence and the transition to young adulthood [35-38]; 4) recruiting a raciallydiverse sample from large metropolitan area to advance the science on racial/ethnic variations in AMH among young women [25-27]; and 5) testing associations between $\mathrm{AMH}$ and a range of physical, mental, reproductive, and behavioral health correlates and confounders most relevant to young women.

Regarding procedures for bioassay collection and laboratory analysis, our approach and descriptive $\mathrm{AMH}$ results appear to be consistent with the most recent bioassay studies of blood spot AMH that have included healthy samples of reproductive aged women [24]. Our participants also reported our data collection methods to be highly feasible and acceptable. We initiated the study with a smallerbore lancet based on the rationale this would be more acceptable to our participants, but for some it was difficult to collect enough blood for replicate spots on the filter paper. The use of different lancet $(\mathrm{mm}=30)$ also had good compliance and gave better more consistent blood collection $[39,40]$. The acceptance and feasibility of blood spot collection in this project reflects a growing preference of this collection rather than serum samples for obtaining biological data for biosocial research.

The modest design and resources of our pilot precluded a formal, comprehensive validation study, particularly given that quality of DBS samples was a challenge for bioassay data collection and analysis given less-than-complete collection circles filled on the filter paper and given our young sample experienced few pregnancies during the study period. Thus, we were not able to compare AMH levels to time to pregnancy or validate against other more invasive fertility measures, such as the gold standard antral follicle count. However, we did collect a robust series of indirect reproductive function measures appropriate for an adolescent and young adult sample, including detailed monthly menstrual cycle data. Our findings that higher AMH levels were associated with longer length and irregular patterned menstrual cycles were not surprising. We also found that lower AMH levels were associated with current contraceptive use, but not ever use, which is also generally consistent with prior research [41]. Yet, in our study AMH levels were not associated with any of the other known physical, mental and behavioural health correlates of AMH that have been found in other studies and were of interest here. Furthermore, we did not see differences in $\mathrm{AMH}$ levels by age or by race/ethnicity, as we would have hypothesized. These null findings may be due to insufficient subsample sizes, which limited our statistical power to explore our various correlate indicators. Additionally, our sample comprised a relatively healthy within a confined age range who were recruited from nonclinical sites in a single southeaster city. Taken together, the utility of blood spot AMH measure for community- and population-based adolescent reproductive health and family planning research remains unclear.

Our findings, coupled with those of Steiner, suggest that public health and social science researchers should use caution when considering $\mathrm{AMH}$ as a potential biological indicator in communityand population-based research for adolescent reproductive health and family planning. More rigorous studies are needed to determine the scientific utility of this bioassay across the early reproductive life course. Study designs with larger and more generalizable samples and intensive repeated measurements of $\mathrm{AMH}$, other fertility indicators, time-to-pregnancy, and relevant health and psychosocial confounders over longer study periods are required to assess AMH's validity for a young healthy population.

Perhaps most importantly, our experience has brought into focus the meaning of a biological measure of fecundity and its interpretation within the context of "unintended pregnancy" and disparities in family planning. Among the most salient questions raised are: 1) What is the usefulness of identifying sociodemographic differentials in $\mathrm{AMH}$ levels and in fecundity generally across racial/ethnic and other groups of young women not desiring or attempting pregnancy? 2) What are the potential implications, undue consequences, and harms of labelling and subsequently targeting "biological risk" of unintended pregnancy, especially when our field is grappling with the notion of unintended pregnancy itself not as a public health problem but rather as a stigmatizing social construct that may undermine the ways in which structures and systems should support the range of reproductive needs and goals of all women, regardless of age, race, class, etc? 3) What is the complex interplay of biological, behavioural, and social factors that likely shape $\mathrm{AMH}$, biological fecundity, and pregnancy intentions and outcomes and how do these intersectional factors shape disparities in family planning? 4) What would even be the goals of clinical, public health and policy interventions and programs designed to "address" 
AMH and biological fecundity in the context of adolescent reproductive health? And 5) Given the above concerns, are biomarkers related to fertility cost-effective or a prudent use of research resources for population- and community-based studies of adolescent reproductive health?

In conclusion, this analysis and our larger research program is attempting to advance innovative scientific methods to address the dynamic, interactive biosocial processes of reproductive health across the life course and across diverse populations. Whether AMH as marker of reproductive health has research utility will require further investigation. Our findings suggest that public health and social science researchers interested in biosocial methods using AMH approach their designs with great care, ethical consideration, and with a reproductive justice and human rights frame. Ultimately, we hope that the modest findings and critical thinking resulting from this work can be used to advance the conversation toward people-centred and more holistic reproductive health services, programs, and policies required to improve family planning outcomes and inequities in the U.S. and beyond.

\section{Funding information}

This work was supported by National Institute of Child Health and Human Development grant \#K01HD080722-01A1 (KSH).

\section{References}

1. Finer LB (2010) Unintended pregnancy among U.S. adolescents: accounting for sexual activity. J Adolesc Health 47: 312-314. [Crossref]

2. Finer LB, Henshaw SK (2006) Disparities in rates of unintended pregnancy in the United States, 1994 and 2001. Perspect Sex Reprod Health 38: 90-96. [Crossref]

3. Control CfD (1999) Prevention, Control CfD, Prevention. Achievements in public health, 1900-1999: family planning. MMWR Morb Mortal Wkly Rep 48: 1073-1080. [Crossref]

4. Bongaarts J (1978) A framework for analyzing the proximate determinants of fertility. Population Development Review 4: 105-132.

5. Harris KM (2010) An integrative approach to health. Demography 47: 1-22. [Crossref]

6. Roux AVD (2007) Integrating social and biologic factors in health research: a systems view. Ann Epidemiol 17: 569-574. [Crossref]

7. Davis K, Blake J. Social structure and fertility: An analytic framework. Economic development and cultural change 1956;4(3):211-235. [Crossref]

8. Harris KM, McDade TW (2018) The biosocial approach to human development, behavior, and health across the life course. $R S F 4: 2-26$. [Crossref]

9. Harris KM, Schorpp KM (2018) Integrating biomarkers in social stratification and health research. Annu Rev Sociol 44: 361-386. [Crossref]

10. Harris KM (2012) The Add Health Study: design and accomplishments. Avaliable from: $\quad$ https://www.cpc.unc.edu/projects/addhealth/documentation/guides/ DesignPaperWIIV.pdf

11. Barber JS, Kusunoki Y, Gatny HH (2011) Design and implementation of an online weekly journal to study unintended pregnancies. Vienna Yearb Popul Res 9: 327-334. [Crossref]

12. Ferrell RJ, Sowers M (2010) Longitudinal, epidemiologic studies of female reproductive aging. Ann N Y Acad Sci 1204: 188-197. [Crossref]

13. Dewailly D, Andersen CY, Balen A, Broekmans F, Dilaver N, et al. (2014) The physiology and clinical utility of anti-Mullerian hormone in women. Hum Reprod Update 20: 370-385. [Crossref]

14. Broer SL, Broekmans FJ, Laven JS, Fauser BC (2014) Anti-Mullerian hormone: ovarian reserve testing and its potential clinical implications. Hum Reprod Update 20: 688-701. [Crossref]

15. McDade TW, Woodruff TK, Huang YY, Funk WE, Prewitt M, et al. (2012) Quantification of anti-Mullerian hormone (AMH) in dried blood spots: validation of a minimally invasive method for assessing ovarian reserve. Hum Reprod 27: 2503-2508. [Crossref]
16. Fleming R, Seifer DB, Frattarelli JL, Ruman J (2015) Assessing ovarian response: antral follicle count versus anti-Mullerian hormone. Reprod Biomed Online 31: 486496. [Crossref]

17. Freeman EW, Sammel MD, Lin H, Gracia CR (2012) Anti-mullerian hormone as a predictor of time to menopause in late reproductive age women. J Clin Endocrinol Metab 97: 1673-1680. [Crossref]

18. Hagen CP, Aksglaede L, Sorensen K, Mouritsen A, Andersson AM, et al. (2012) Individual serum levels of anti-Mullerian hormone in healthy girls persist through childhood and adolescence: a longitudinal cohort study. Hum Reprod 27: 861-886. [Crossref]

19. Hagen CP, Vestergaard S, Juul A, Skakkebaek NE, Andersson AM, et al. (2012) Low concentration of circulating antimullerian hormone is not predictive of reduced fecundability in young healthy women: a prospective cohort study. Fertil Steril 98 1602-1608 e2. [Crossref]

20. Hagen CP, Aksglaede L, Sorensen K, Main KM, Boas M, et al. (2010) Serum levels of anti-Mullerian hormone as a marker of ovarian function in 926 healthy females from birth to adulthood and in 172 Turner syndrome patients. J Clin Endocrinol Metab 95: 5003-5010. [Crossref]

21. Hagen CP, Sorensen K, Anderson RA, Juul A (2012) Serum levels of antimullerian hormone in early maturing girls before, during, and after suppression with $\mathrm{GnRH}$ agonist. Fertil Steril 98: 1326-1330. [Crossref]

22. Pinola P, Morin-Papunen LC, Bloigu A, Puukka K, Ruokonen A, et al. (2014) AntiMullerian hormone: correlation with testosterone and oligo- or amenorrhoea in female adolescence in a population-based cohort study. Hum Reprod 29: 2317-2325. [Crossref]

23. Steiner AZ, Herring AH, Kesner JS, Meadows JW, Stanczyk FZ, et al. (2011) Antimullerian hormone as a predictor of natural fecundability in women aged 30-42 years. Obstet Gynecol 117: 798-804. [Crossref]

24. Steiner AZ, Pritchard D, Stanczyk FZ, Kesner JS, Meadows JW, et al. (2017) Association Between Biomarkers of Ovarian Reserve and Infertility Among Older Women of Reproductive Age. JAMA 318: 1367-1376. [Crossref]

25. Elchuri SV, Patterson BC, Brown MR, Buchanan I, Mertens AC, et al. (2015) AntiMullerian hormone levels in American girls by age and race/ethnicity. $J$ Pediat Endocrinol Metab 28: 189-93. [Crossref]

26. Bleil ME, Gregorich SE, Adler NE, Sternfeld B, Rosen MP, et al. (2014) Race/ethnic disparities in reproductive age: an examination of ovarian reserve estimates across four race/ ethnic groups of healthy, regularly cycling women. Fertil Steril 101: 199-207. [Crossref]

27. Tal R, Seifer DB (2013) Potential mechanisms for racial and ethnic differences in antimullerian hormone and ovarian reserve. Int J Endocrinol: 818912. [Crossref]

28. Kumar A, Kalra B, Patel A, McDavid L, Roudebush WE (2010) Development of a second generation anti-Müllerian hormone (AMH) ELISA. J Immunol Methods 362 51-59. [Crossref]

29. Harris PA, Taylor R, Minor BL, Elliott V, Fernandez M, et al. (2019) The REDCap consortium: Building an international community of software platform partners. $J$ Biomed Inform 95: 103208. [Crossref]

30. Harris PA, Taylor R, Thielke R, Payne J, Gonzalez N, et al. (2009) Research electronic data capture (REDCap) - a metadata-driven methodology and workflow process for providing translational research informatics support. J Biomed Inform 42: 377-381. [Crossref]

31. Hall KS, Beauregard JL, Rentmeester ST, Livingston M, Harris KM (2019) Adverse life experiences and risk of unintended pregnancy in adolescence and early adulthood: Implications for toxic stress and reproductive health. SSM Popul Health 7: 100344. [Crossref]

32. Hall KS, Kusunoki Y, Gatny H, Barber J (2014) The risk of unintended pregnancy among young women with mental health symptoms. Soc Sci Med 100: 62-71. [Crossref]

33. McNeely C, Blanchard J (2010) The teen years explained: A guide to healthy adolescent development. Jayne Blanchard.

34. Ben-Shlomo Y, Kuh D (2002) A life course approach to chronic disease epidemiology: conceptual models, empirical challenges and interdisciplinary perspectives. Int $J$ Epidemiol 31: 285-293.

35. Dyl J (2013) Helping teens cope with stress. Avlaible from: http://www.lifespan.org/ services/childhealth/parenting/teen-stress.html

36. Moos MK, Dunlop AL, Jack BW, Nelson L, Coonrod DV, et al. (2008) Healthier women, healthier reproductive outcomes: recommendations for the routine care of all women of reproductive age. Am J Obstet Gynecol 199: S280-S289. [Crossref]

37. Su X, Carlson BF, Wang X, Li X, Zhang Y, et al. (2018) Dried blood spots: An evaluation of utility in the field. J Infect Public Health 11: 373-376. [Crossref] 
Hall KS (2020) A pilot study for exploring blood spot anti-mullerian hormone for population-based adolescent reproductive health research

38. Bingley PJ, Rafkin LE, Matheson D, Steck AK, Yu L, et al. (2015) Use of dried capillary blood sampling for islet autoantibody screening in relatives: a feasibility study. Diabetes Technol Ther 17: 867-871. [Crossref]

39. Amer S, James C, Al-Hussaini TK, Mohamed AA (2019) Assessment of Circulating Anti-Mullerian Hormone in Women Using Hormonal Contraception: A Systematic Review. J Womens Health (Larchmt) 29: 100-110. [Crossref]
40. Huffling K (2011) Prenatal-Preconception Environmental Exposure Assessment. Alliance of Nurses for Healthy Environments.

41. Cohen S, Kamarck T, Mermelstein R (1983) A global measure of perceived stress. $J$ Health Soc Behav 24: 385-396. [Crossref]

Copyright: $(02020$ Hall KS. This is an open-access article distributed under the terms of the Creative Commons Attribution License, which permits unrestricted use, distribution, and reproduction in any medium, provided the original author and source are credited. 